\title{
Prognostic Evaluation of Tumor-Stroma Ratio in Patients with Early Stage Cervical Adenocarcinoma Treated by Surgery
}

\author{
Tip Pongsuvareeyakul ${ }^{1}$, Surapan Khunamornpong ${ }^{1 *}$, Jongkolnee Settakorn ${ }^{1}$, \\ Kornkanok Sukpan ${ }^{1}$, Prapaporn Suprasert ${ }^{2}$, Suthida Intaraphet ${ }^{3}$, Sumalee \\ Siriaunkgul ${ }^{1}$
}

\begin{abstract}
Background: The tumor-stroma ratio (TSR) represents the percentage of neoplastic cell components compared to the combined area of neoplastic cells and the surrounding tumor-induced stroma. A low TSR (predomination of stromal component) has been demonstrated to be an independent adverse prognostic factor in cancers of several organs. In cervical carcinoma patients, TSR has been evaluated in only one previous study with different histological types. The present study aimed to assess the prognostic value of TSR in early stage cervical cancer patients with adenocarcinoma histology only. Materials and Methods: Histological slides of patients with early stage (IB-IIA) cervical adenocarcinoma who underwent surgical treatment between January 2003 and December 2011 were reviewed. Patients who had received preoperative chemotherapy were excluded. TSR was categorized as low $(<50 \%)$ and high $(\geq \mathbf{5 0 \%})$. Correlations between TSR and clinicopathological variables were evaluated. Prognostic values of TSR and other variables were estimated using Cox's regression. Results: Of 131 patients; $38(29.0 \%)$ had low TSR and $93(71.0 \%)$ had high TSR. The patients with low TSR had significantly higher proportions of deep cervical stromal invasion (outer third of wall, $p=0.011$; residual stroma less than $3 \mathrm{~mm}$, $\mathrm{p}=\mathbf{0 . 0 0 8}$ ) and parametrial involvement $(\mathrm{p}=\mathbf{0 . 0 2 6})$. Compared to the patients with high TSR, those with low TSR tended to have lower 5-year disease-free survival rate $(83.8 \%$ versus $88.9 \%)$ and overall survival rate $(85.6 \%$ versus $90.3 \%$ ), although the differences were not statistically significant. Low TSR was significantly associated with decreased overall survival in univariate analysis (HR 2.7; 95\% CI 1.0-7.0; $p=0.041$ ), but not in multivariate analysis. TSR was not significantly associated with decreased disease-free survival. Conclusions: Low TSR is associated with decreased overall survival in patients with early stage cervical adenocarcinoma treated by surgery. However, it was not found to be an independent prognostic predictor in this study.
\end{abstract}

Keywords: Cervical cancer - adenocarcinoma - prognosis - tumor-stroma ratio

Asian Pac J Cancer Prev, 16 (10), 4363-4368

\section{Introduction}

Cervical cancer is the third most common cancers worldwide, with the large majority of patients living in low-income or developing countries (Jemal et al., 2011). Cervical cancer accounts for $11 \%$ of female cancer death in developing countries and is a leading cause of cancer death among women in the Asia-Pacific region (Jemal et al., 2011; Obel et al., 2014). Histologically, squamous cell carcinoma is the most common type of cervical cancer. Adenocarcinoma is the second most common histological type and makes up approximately $20 \%$ of cervical carcinoma cases, with increasing occurrence in recent years (Gien et al., 2010). Most of early stage cervical cancer patients can be successfully treated by radical surgery and pelvic lymphadenectomy. Histological examination of the resected specimens provides useful prognostic information, which helps to justify adjuvant therapy, such as pelvic lymph node metastasis, positive surgical margins, and parametrial involvement (Hongladaromp et al., 2014).

Cancercells exist in a highly complex microenvironment of the surrounding stroma (Li et al., 2007). Both the neoplastic cell component and the associated stroma interact with each other, and the stromal component has an important role in tumor progression and metastasis (Pietras and Ostman, 2010). Tumor-stroma ratio (TSR) is a recently described histological feature of cancer with promising prognostic potential (Mesker et al., 2007). TSR represents an estimation of the percentage between the neoplastic cell component compared to the combined area of neoplastic cells and tumor-associated stroma. Low TSR has been demonstrated to be an adverse prognostic features in several types of cancer (Mesker et al., 2007; West et al., 2010; de Kruijf et al., 2011; Wang et al., 2012; Dekker et al., 2013). Evaluation of TSR is simple and

${ }^{1}$ Department of Pathology, ${ }^{2}$ Department of Obstetrics and Gynecology, Faculty of Medicine, Chiang Mai University, Chiang Mai, ${ }^{3}$ Boromarajonani College of Nursing, Khon Kaen, Thailand *For correspondence: skhunamo@yahoo.com 
Tip Pongsuvareeyakul et al

based on routine histological material without the need for additional special techniques.

In cervical cancer, there has been only one previous TSR study to our knowledge. The study demonstrated that TSR was an independent prognostic factor in early stage cervical cancer patients (Liu et al., 2014). However, that study was based on a series of cervical carcinoma with different histological types. As the prognostic value of clinicopathological features in cervical carcinoma may not be uniform across different histological types (Intaraphet et al., 2014), it is uncertain whether the prognostic value of TSR would be similar in cervical adenocarcinoma. This study was aimed to evaluate, in a series of pure cervical adenocarcinoma, the relationship of TSR with other clinical and histopathological features and its prognostic value in early stage patients.

\section{Materials and Methods}

After receiving approval by the institution Ethics Committee, the records of patients with early stage cervical adenocarcinoma (stage IB-IIA) who underwent radical hysterectomy and pelvic lymphadenectomy in Chiang Mai University Hospital between January 2003 and December 2011 were retrieved from the Surgical Pathology files of the Department of Pathology. Exclusion criteria included patients with mixed adenocarcinoma and other histological types, patients who received preoperative (neoadjuvant) chemotherapy, and patients who died within 30 days postoperatively.

Hematoxylin and eosin-stained sections of cervical adenocarcinoma were reviewed by one pathologist (T.P.) to confirm the cervical origin and the histological type. Pathological data including tumor size, histological grade, depth of stromal invasion, parametrial involvement,

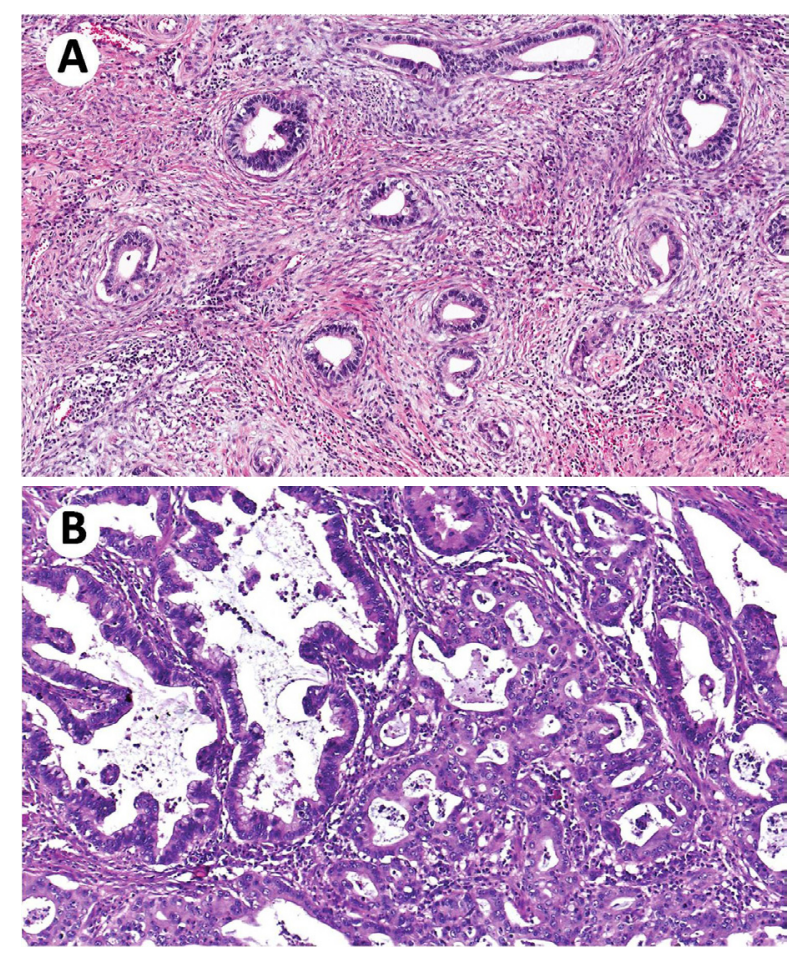

Figure 1. Tumor-Stroma Ratio (TSR) in Cervical Adenocarcinoma. A) Low TSR, B) High TSR surgical margin status, lymphovascular space invasion (LVSI), and lymph node metastasis were obtained from the pathology records, all of which were reported by a team of gynecological pathologists (S.S., S.K., J.S, and K.S.). Clinical data including patient age, FIGO stage, postoperative adjuvant treatment modalities, and followup outcomes were retrieved from the medical records and the civil registration databases in all patients. Regarding the treatment policy, postoperative adjuvant treatment was justified in patients with high-risk parameters (i.e. lymph node metastasis, positive surgical margin, or parametrial involvement) or in patients with at least 2 intermediate risk factors (i.e. tumor size $\geq 4 \mathrm{~cm}$, extensive LVSI, or deep stromal invasion).

The deepest area of invasion of tumor in each case was identified using a low-power magnification of microscope $(x 40)$ by one pathologist (T.P.). The focus with the largest proportion of tumor-associated stroma was selected for TSR estimation. This stromal component was characterized by fibroblastic proliferation of variable degrees within loose or myxoid stroma or collagenous background. TSR represented the percentage of the area of neoplastic cell component compared to the combined area of neoplastic cells and associated stromal component, excluding mucin collections and necrotic material. TSR was scored within a medium-power field area (x100 magnification or $2.0 \mathrm{~mm}$ in diameter) into 2 categories using a 50\% cut-off value as described previously (Mesker et al., 2007; Liu et al., 2014): low TSR ( $<50 \%$ or stromarich) and high TSR ( $\geq 50 \%$ or stroma-poor) (Figure 1). Additional characteristics of stromal inflammatory response in the stroma were recorded separately. TSR was independently evaluated by two pathologists (T.P.

\section{A}

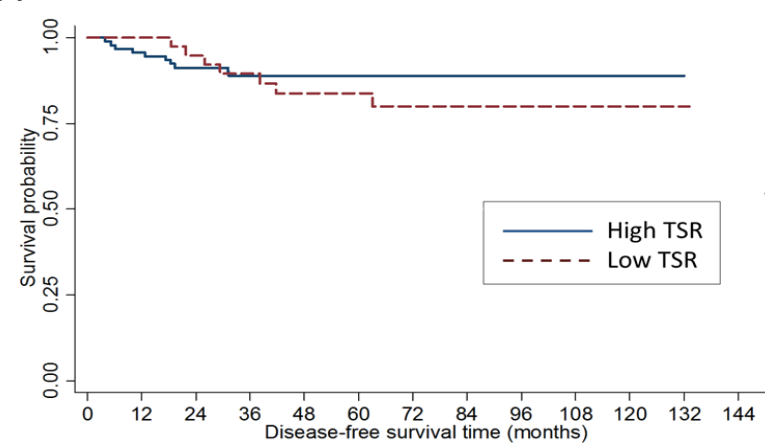

B

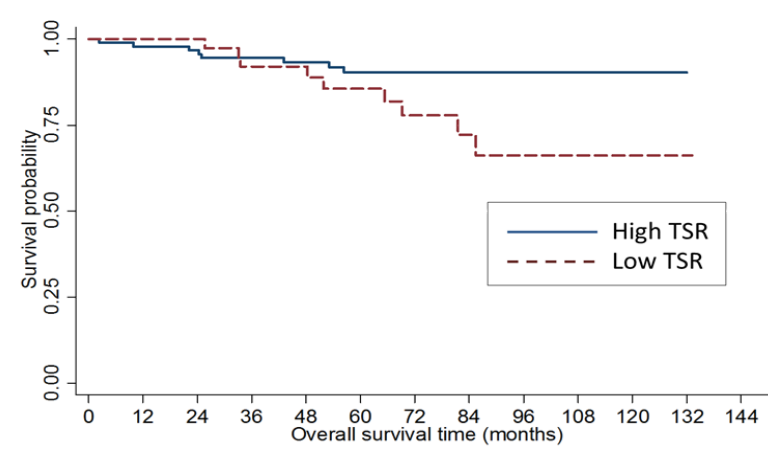

Figure 2. Kaplan-Meier Plots for Survival Stratified by Tumor-Stroma Ratio. A) Disease-free survival, B) Overall survival 
and S.K.) blinded to the clinical outcomes. In cases with discordant TSR results, both pathologists reached a consensus final score by examination of the slides together using a mutiheaded microscope.

All data were analyzed using STATA version 11 (StataCorp LP, college station, TX, USA). The associations of TSR and other clinicopathological parameters were assessed using the exact probability test. Interobserver variability was analyzed using the Cohen's kappa coefficient. The Kaplan-Meier method and log-rank test were used for the analysis and comparison of survival curves. Cox's regression models were used to perform univariable and multivariable analysis of disease-free survival and overall survival. Prognostic predictors with a $\mathrm{p}$ value of less than 0.25 in the univariable analysis were further evaluated in the multivariable analysis. A $\mathrm{p}$ value of less than 0.05 was considered statistically significant. Disease-free survival was defined as the time period between the date of surgery until disease recurrence (locoregional recurrence or distant metastasis), the last
Tumor-stroma Ratio in Early Stage Cervical Adenocarcinoma follow-up, or censoring. Overall survival was defined as the time period between the date of surgery until death from any cause, the last follow-up, or censoring.

\section{Results}

A total of 157 cases of early stage (IB-IIA) cervical adenocarcinoma were retrieved from the Surgical Pathology files. Twenty-six patients (16.6\%) who received pre-operative chemotherapy were excluded. TSR was evaluated in the remaining 131 cases. The median age of the patients was 45 years (range 32-85 years). Of 131 cases, 93 cases $(71.0 \%)$ were categorized as high TSR and 38 cases $(29.0 \%)$ as low TSR. The agreement for TSR evaluation between the two pathologists was reached in 119 cases (90.8\%) with the Cohen's Kappa coefficient of 0.78 which indicated substantial interobserver agreement. The clinical and pathological data of patients stratified by TSR are presented in Table 1. Patients with low TSR had significantly higher proportions of invasion into

Table 1. Clinical and Pathological Data of 131 Patients with Early Stage Cervical Adenocarcinoma Stratified by Tumor-stroma Ratio (TSR)

\begin{tabular}{|c|c|c|c|c|}
\hline Feature & Total No. $(\%), \mathrm{n}=131$ & Low TSR No. $(\%), \mathrm{n}=38$ & High TSR No. $(\%), \mathrm{n}=93$ & $\mathrm{p}$ value \\
\hline \multicolumn{5}{|l|}{ Age } \\
\hline$\leq 45$ years & $75(57.3)$ & $19(50.0)$ & $56(60.2)$ & \multirow[t]{2}{*}{0.332} \\
\hline$>45$ years & $56(42.7)$ & $19(50.0)$ & $37(39.8)$ & \\
\hline \multicolumn{5}{|l|}{ Stage } \\
\hline IB1 & $101(77.1)$ & $25(65.8)$ & $76(81.7)$ & \multirow[t]{2}{*}{0.066} \\
\hline IB2-IIA2 & $30(22.9)$ & $13(34.2)$ & $17(18.3)$ & \\
\hline \multicolumn{5}{|l|}{ Size } \\
\hline$\leq 4.0 \mathrm{~cm}$ & $113(86.3)$ & $31(81.6)$ & $82(88.2)$ & \multirow[t]{2}{*}{0.402} \\
\hline$>4.0 \mathrm{~cm}$ & $18(13.7)$ & $7(18.4)$ & $11(11.8)$ & \\
\hline \multicolumn{5}{|l|}{ Histologic grade } \\
\hline Grade $1-2$ & $114(87.0)$ & $32(84.2)$ & $82(88.2)$ & \multirow[t]{2}{*}{0.572} \\
\hline Grade 3 & $17(13.0)$ & $6(15.8)$ & $11(11.8)$ & \\
\hline \multicolumn{5}{|c|}{ Inflammatory response to tumor } \\
\hline Mild & $31(23.7)$ & $8(21.0)$ & $23(24.7)$ & \multirow[t]{2}{*}{0.821} \\
\hline Moderate to marked & $100(76.3)$ & $30(79.0)$ & $70(75.3)$ & \\
\hline \multicolumn{5}{|c|}{ Predominant inflammatory cells } \\
\hline Lymphocytes/plasma cells & $121(92.4)$ & $34(89.5)$ & $87(93.5)$ & \multirow[t]{2}{*}{0.475} \\
\hline Neutrophils/eosinophils & $10(7.6)$ & $4(10.5)$ & $6(6.5)$ & \\
\hline \multicolumn{5}{|l|}{ Fraction of stromal invasion } \\
\hline Inner to middle third & $54(41.5)$ & $9(23.7)$ & $45(48.9)$ & \multirow[t]{2}{*}{0.011} \\
\hline Outer third & $76(58.5)$ & $29(76.3)$ & $47(51.1)$ & \\
\hline \multicolumn{5}{|l|}{ Tumor-free residual stroma } \\
\hline$\geq 3.0 \mathrm{~mm}$ & $86(65.6)$ & $18(47.4)$ & $68(73.1)$ & \multirow[t]{2}{*}{0.008} \\
\hline$<3.0 \mathrm{~mm}$ & $45(34.4)$ & $20(52.6)$ & $25(26.9)$ & \\
\hline \multicolumn{5}{|l|}{ Parametrial involvement } \\
\hline Negative & $112(85.5)$ & $28(73.7)$ & $84(90.3)$ & \multirow[t]{2}{*}{0.026} \\
\hline Positive & $19(14.5)$ & $10(26.3)$ & $9(9.7)$ & \\
\hline \multicolumn{5}{|l|}{ Vaginal margin } \\
\hline Negative & $123(93.9)$ & $34(89.5)$ & $89(95.7)$ & \multirow[t]{2}{*}{0.229} \\
\hline Positive & $8(6.1)$ & $4(10.5)$ & $4(4.3)$ & \\
\hline \multicolumn{5}{|l|}{ Lymphovascular invasion } \\
\hline Negative & $62(47.3)$ & $13(34.2)$ & $49(52.7)$ & \multirow[t]{2}{*}{0.082} \\
\hline Positive & $69(52.7)$ & $25(65.8)$ & $44(47.3)$ & \\
\hline \multicolumn{5}{|l|}{ Lymph node metastasis } \\
\hline Negative & $106(80.9)$ & $28(73.7)$ & $78(83.9)$ & \multirow[t]{2}{*}{0.221} \\
\hline Positive & $25(19.1)$ & $10(26.3)$ & $15(16.1)$ & \\
\hline \multicolumn{5}{|l|}{ Adjuvant therapy } \\
\hline No & $89(67.9)$ & $19(50.0)$ & $70(75.3)$ & \multirow[t]{2}{*}{0.007} \\
\hline Yes & $42(32.1)$ & $19(50.0)$ & $23(24.7)$ & \\
\hline
\end{tabular}


Table 2. Univariable and Multivariable Analysis of Clinical and Pathological Features on Disease-free Survival and Overall Survival

\begin{tabular}{|c|c|c|c|c|c|c|c|c|}
\hline \multirow[t]{3}{*}{ Feature } & \multicolumn{4}{|c|}{ Disease-free survival } & \multicolumn{4}{|c|}{ Overall survival } \\
\hline & \multicolumn{2}{|c|}{ Univariable analysis } & \multicolumn{2}{|c|}{ Multivariable analysis } & \multicolumn{2}{|c|}{ Univariable analysis } & \multicolumn{2}{|c|}{ Multivariable analysis } \\
\hline & $\begin{array}{c}\mathrm{HR} \\
(95 \% \mathrm{CI})\end{array}$ & $\mathrm{p}$ value & $\begin{array}{c}\mathrm{HR} \\
(95 \% \mathrm{CI})\end{array}$ & $\mathrm{p}$ value & $\begin{array}{c}\text { HR } \\
(95 \% \mathrm{CI})\end{array}$ & $\mathrm{p}$ value & $\underset{(95 \% \mathrm{CI})}{\mathrm{HR}} \mathrm{p}$ & value \\
\hline \multicolumn{9}{|l|}{ Age } \\
\hline$\leq 45$ years & 1 & 0.323 & - & - & 1 & 0.110 & 1 & 0.009 \\
\hline$>45$ years & $1.6(0.6-4.2)$ & & - & & $2.2(0.7-5.8)$ & & $7.7(1.7-35.7)$ & \\
\hline \multicolumn{9}{|l|}{ Tumor-stroma ratio } \\
\hline High & 1 & 0.336 & - & - & 1 & 0.041 & 1 & 0.692 \\
\hline Low & $1.6(0.6-4.2)$ & & - & & $2.7(1.0-7.0)$ & & $1.3(0.4-4.0)$ & \\
\hline \multicolumn{9}{|l|}{ Stage } \\
\hline IB1 & 1 & 0.016 & 1 & 0.771 & 1 & 0.380 & - & - \\
\hline IB2-IIA2 & $3.2(1.3-8.4)$ & & $1.2(0.3-4.2)$ & & $1.6(0.6-4.5)$ & - & & \\
\hline \multicolumn{9}{|l|}{ Size } \\
\hline$\leq 4.0 \mathrm{~cm}$ & 1 & 0.008 & 1 & 0.124 & 1 & 0.490 & - & - \\
\hline$>4.0 \mathrm{~cm}$ & $3.9(1.4-10.5)$ & & $2.7(0.8-9.4)$ & & $1.6(0.5-5.4)$ & - & & \\
\hline \multicolumn{9}{|l|}{ Histologic grade } \\
\hline Grade $1-2$ & 1 & 0.554 & - & - & 1 & 0.252 & - & - \\
\hline Grade 3 & $1.5(0.4-5.1)$ & & - & & $1.9(0.6-5.9)$ & - & & \\
\hline \multicolumn{9}{|c|}{ Inflammatory response to tumor } \\
\hline Mild & 1 & 0.613 & - & - & 1 & 0.485 & - & - \\
\hline Moderate to marked & $0.8(0.3-2.2)$ & & - & & $1.6(0.5-5.4)$ & - & & \\
\hline \multicolumn{9}{|l|}{ Predominant inflammatory cells } \\
\hline Lymphocytes/plasma cells & 1 & 0.101 & 1 & 0.209 & 1 & 0.524 & - & - \\
\hline Neutrophils/eosinophils & $2.8(0.8-9.9)$ & & $2.4(0.6-9.1)$ & & $1.6(0.4-7.1)$ & - & & \\
\hline \multicolumn{9}{|l|}{ Fraction of stromal invasion } \\
\hline Inner to middle third & 1 & 0.041 & 1 & 0.459 & 1 & 0.028 & 1 & 0.868 \\
\hline Outer third & $3.7(1.1-12.8)$ & & $1.8(0.4-9.4)$ & & $4.1(1.2-14.2)$ & & $1.2(0.2-6.3)$ & \\
\hline \multicolumn{9}{|l|}{ Tumor-free residual stroma } \\
\hline$\geq 3.0 \mathrm{~mm}$ & 1 & 0.025 & 1 & 0.909 & 1 & 0.003 & 1 & 0.280 \\
\hline$<3.0 \mathrm{~mm}$ & $3.0(1.2-8.0)$ & & $0.9(0.3-3.3)$ & & $4.5(1.7-12.3)$ & & $2.1(0.5-8.5)$ & \\
\hline \multicolumn{9}{|l|}{ Parametrial involvement } \\
\hline Negative & 1 & 0.224 & 1 & 0.308 & 1 & 0.121 & 1 & 0.252 \\
\hline Positive & $2.0(0.6-6.2)$ & & $0.5(0.1-2.0)$ & & $2.4(0.8-7.5)$ & & $0.4(0.1-2.0)$ & \\
\hline \multicolumn{9}{|l|}{ Vaginal margin } \\
\hline Negative & 1 & 0.250 & 1 & 0.357 & 1 & 0.126 & 1 & 0.979 \\
\hline Positive & $2.4(0.5-10.5)$ & & $2.4(0.4-14.7)$ & & $3.2(0.7-14.3)$ & & $1.0(0.1-7.4)$ & \\
\hline \multicolumn{9}{|l|}{ Lymphovascular invasion } \\
\hline Negative & 1 & 0.007 & 1 & 0.225 & 1 & 0.006 & 1 & 0.040 \\
\hline Positive & $7.6(1.7-33.1)$ & & $2.7(0.5-13.7)$ & & $7.8(1.8-34.3)$ & & $5.2(1.1-25.5)$ & \\
\hline \multicolumn{9}{|l|}{ Lymph node metastasis } \\
\hline Negative & 1 & 0.003 & 1 & 0.454 & 1 & 0.002 & 1 & 0.011 \\
\hline Positive & $4.2(1.6-10.9)$ & & $1.5(0.5-4.4)$ & & $4.7(1.8-12.2)$ & & $7.7(1.6-37.1)$ & \\
\hline \multicolumn{9}{|l|}{ Adjuvant therapy } \\
\hline No & 1 & $<0.001$ & 1 & 0.006 & 1 & 0.014 & 1 & 0.385 \\
\hline Yes & $18.5(4.2-81.1)$ & & $9.2(1.9-44.3)$ & & $3.4(1.3-8.9)$ & & $1.8(0.5-6.7)$ & \\
\hline
\end{tabular}

HR: Hazard ratio; CI: Confidence interval

the outer third of cervical stroma $(\mathrm{p}=0.011)$, tumor-free residual stroma less than $3 \mathrm{~mm}(\mathrm{p}=0.008)$, and parametrial involvement $(\mathrm{p}=0.026)$.

The median follow-up time was 73 months (range 2-133 months). Seventeen patients had tumor recurrence; 11 with locoregional recurrence and 6 with distant metastasis. Seventeen patients died; 11 had documented progression of the disease. Due to the low rate of recurrence and death in this study, the median survival time for disease recurrence and death was not reached. Compared to the patients with high TSR, those with low TSR tended to have a lower 5-year disease-free survival rate $(83.8 \%$ versus $88.9 \%, \mathrm{p}=0.497)$ and a lower 5 -year overall survival rate ( $85.6 \%$ versus $90.3 \%, \mathrm{p}=0.151)$. The Kaplan-Meier plots for survivals are shown in Figure 2. The patients with low TSR had significantly lower overall survival than those with high TSR $(\mathrm{p}=0.033)$. The patients with low TSR also had a trend toward lower disease-free survival but without statistical significance $(\mathrm{p}=0.331)$.

Table 2 shows the results of Cox univariable and multivariable analysis of the clinical and pathological features on disease-free survival and overall survival. In univariable analysis, low TSR was significantly associated with decreased overall survival (HR 2.7; 95\% CI 1.0-7.0; $\mathrm{p}=0.041$ ), but no statistical significance was observed for disease-free survival.

In multivariable analysis, TSR was not an independent prognostic predictor for either overall or disease-free survival. Patient age of more than 45 years (HR 7.7; 95\% CI 1.7-35.7; p=0.009), positive LVSI (HR 5.2; 95\% CI 1.1-25.5; $\mathrm{p}=0.040$ ), and lymph node metastasis (HR 7.7; 95\% CI 1.6-37.1; $\mathrm{p}=0.011$ ) were independent adverse predictors for overall survival, whereas postoperative adjuvant therapy (HR 9.2; 95\% CI 1.9-44.3; p=0.006) was an independent predictor for decreased disease-free survival. 


\section{Discussion}

TSR was first described as an independent prognostic feature in colorectal cancer (Mesker et al., 2007), and it has now been extended to other types of cancer including cervical cancer (Liu et al., 2014). TSR reflects the amount of the stromal component surrounding cancer cells. Cancer cells can induce alterations of stromal phenotypes and functions which promote their aggressive potential (De Wever and Mareel, 2003). The tumor-induced stroma is composed mainly of mesenchymal cells, including fibroblasts and myofibroblasts, within the extracellular matrix that contains structural proteins and signaling molecules (Li et al., 2007). Other cellular populations in the stromal microenvironment of the tumor include vascular endothelial cells, smooth muscle cells, and inflammatory or immune cells (Li et al., 2007; Zhu et al., 2013). These cells contribute to the accumulation of various types of cytokines and growth factors in the extracellular matrix. In our previous studies of cervical squamous cell carcinoma, stromal reaction and the inflammatory response were interrelated and associated with other prognostic pathological variables (Khunamornpong et al., 2013). These changes may also provide some prognostic contribution when considered in combination with the invasive pattern of carcinoma (Khunamornpong et al., 2014).

Cancer-associated fibroblasts (and myofibroblasts) have an important role in tumor aggressiveness. They can induce epithelial to mesenchymal transition of carcinoma cells and produce various growth factors and cytokines, angiogenic molecules, and proteolytic enzymes including matrix metalloproteinases (Wever and Mareel, 2003; Li et al., 2007; Kawashiri et al., 2009; Pietras and Ostman, 2010; Zhu et al., 2013). These properties facilitate tumor growth and promote the local invasion of surrounding tissue as well as increasing metastatic spread. Furthermore, the presence of myofibroblasts may prevent the infiltration of immune cells into tumor and this may contribute to the escape of cancer cells from immune control during the immunoediting process (De Wever and Mareel, 2003; Kim et al., 2007). Therefore, an increase in the proportion of the stromal component may promote an increasing aggressive potential of tumor and may result in poor clinical outcomes (Wever and Mareel, 2003; Li et al., 2007; Kawashiri et al., 2009).

The prognostic value of TSR has been evaluated in several types of cancers of non-gynecological origin including colon, breast, and esophagus, and all these studies reached a similar conclusion for an adverse effect of low TSR on the survival outcomes (Mesker et al., 2007; West et al., 2010; de Kruijf et al., 2011; Wang et al., 2012; Dekker et al., 2013). TSR may also help to make decisions about adjuvant treatment for some specific subgroups of patients with breast cancer (de Kruijf et al., 2011; Dekker et al., 2013). In cervical cancer patients, TSR has been reported to be an independent prognostic variable in one previous study which included 184 cases of early stage cervical carcinoma, $50.5 \%$ of which had squamous histology whereas $46.2 \%$ had adenocarcinoma (Liu et al., 2014). Compared to the patients with high
Tumor-stroma Ratio in Early Stage Cervical Adenocarcinoma

TSR, those with low TSR had significantly lower 5-year disease-free survival rate $(62.2 \%$ versus $88.4 \%, \mathrm{p}=0.001)$ and overall survival rate $(70.3 \%$ versus $92.5 \%, \mathrm{p}=0.001)$ (Liu et al., 2014). This raises a possibility that TSR could be another risk factor in the justification of adjuvant therapy in patients with early stage cervical cancer. The simple approach and the high reproducibility in TSR evaluation, with Kappa values of 0.81 in the previous study (Liu et al., 2014) and 0.78 in the present study, make TSR an interesting histological prognostic variable in cervical cancer.

In the present study which includes 131 patients with early stage pure adenocarcinoma, low TSR was associated with decreased overall survival in univariable analysis. However, the association was not independent in the multivariable analysis, and TSR did not significantly affect the disease-free survival. Our findings suggest that TSR may have less prognostic impact among adenocarcinoma patients than in those with squamous cell carcinoma. This difference may be partly explained by the difference between histological types of cervical cancer which results in different impact of standard prognostic variables between each histological type (Intaraphet et al., 2013; Intaraphet et al., 2014). Nevertheless, low TSR was associated with other well-recognized adverse pathological prognostic features such as deep cervical stromal invasion and parametrial involvement (Park et al., 2010; Biewenga et al., 2011; Kato et al., 2013). Lymph node metastasis and LVSI were independent pathological prognostic predictors for poor overall survival in our study which was similar to the findings in previous studies (Gien et al., 2010; Park et al., 2010; Kato et al., 2013). In this study, postoperative adjuvant therapy was an independent predictor of decreased disease-free survival. This may be due to the fact that the adjuvant treatment was justified in patients with high-risk pathological features (Peters et al., 2000; Biewenga et al., 2011).

The limitation in this study was the small number of patients with tumor recurrence and death, which may be related to the effectiveness of treatment of patients in early stage adenocarcinoma. The small number of cases may reduce the ability of statistical analysis to assess the potential prognostic value of TSR. Recently, a new prognostic classification system of cervical adenocarcinoma based on the characteristics of the invasive growth pattern has been proposed (Diaz De Vivar et al., 2013). This may be a useful prognostic predictor in cervical adenocarcinoma (Roma et al., 2015), and its value should be confirmed in further studies using multivariable analysis on the clinicopathological details.

In conclusion, low TSR is associated with decreased overall survival of patients with cervical adenocarcinoma. However, it does not significantly provide additional prognostic value to the other standard histological variables. In this study, TSR was not found to be an independent prognostic predictor in patients with early stage cervical adenocarcinoma treated by surgery.

\section{Acknowledgements}

This study was supported by 1) the National Research 
Tip Pongsuvareeyakul et al

University Project, under Thailand's Office of the Higher Education Commission; and 2) the Faculty of Medicine, Chiang Mai University. The authors declare that there is no conflict of interest.

\section{References}

Biewenga P, van der Velden J, Mol BW, et al (2011). Prognostic model for survival in patients with early stage cervical cancer. Cancer, 117, 768-76.

de Kruijf EM, van Nes JG, van de Velde CJ, et al (2011). Tumorstroma ratio in the primary tumor is a prognostic factor in early breast cancer patients, especially in triple-negative carcinoma patients. Breast Cancer Res Treat, 125, 687-96.

De Wever O, Mareel M (2003). Role of tissue stroma in cancer cell invasion. $J$ Pathol, 200, 429-47.

Dekker TJ, van de Velde CJ, van Pelt GW, et al (2013). Prognostic significance of the tumor-stroma ratio: validation study in node-negative premenopausal breast cancer patients from the EORTC perioperative chemotherapy (POP) trial (10854). Breast Cancer Res Treat, 139, 371-9.

Diaz De Vivar A, Roma AA, Park KJ, et al (2013). Invasive endocervical adenocarcinoma: proposal for a new patternbased classification system with significant clinical implications: a multi-institutional study. Int J Gynecol Pathol, 32, 592-601.

Gien LT, Beauchemin MC, Thomas G (2010). Adenocarcinoma: a unique cervical cancer. Gynecol Oncol, 116, 140-6.

Hongladaromp W, Tantipalakorn C, Charoenkwan K, et al (2014). Locoregional spread and survival of stage IIA1 versus stage IIA2 cervical cancer. Asian Pac J Cancer Prev, 15, 887-90.

Intaraphet S, Kasatpibal N, Siriaunkgul S, et al (2013). Prognostic impact of histology in patients with cervical squamous cell carcinoma, adenocarcinoma and small cell neuroendocrine carcinoma. Asian Pac J Cancer Prev, 14, 5355-60.

Intaraphet S, Kasatpibal N, Sogaard M, et al (2014). Histological type-specific prognostic factors of cervical small cell neuroendocrine carcinoma, adenocarcinoma, and squamous cell carcinoma. Onco Targets Ther, 7, 1205-14.

Jemal A, Bray F, Center MM, et al (2011). Global cancer statistics. CA Cancer J Clin, 61, 69-90.

Kato T, Watari H, Takeda M, et al (2013). Multivariate prognostic analysis of adenocarcinoma of the uterine cervix treated with radical hysterectomy and systematic lymphadenectomy. $J$ Gynecol Oncol, 24, 222-8.

Kawashiri S, Tanaka A, Noguchi N, et al (2009). Significance of stromal desmoplasia and myofibroblast appearance at the invasive front in squamous cell carcinoma of the oral cavity. Head Neck, 31, 1346-53.

Khunamornpong S, Lekawanvijit S, Settakorn J, et al (2014). Prognostic model in patients with early-stage squamous cell carcinoma of the uterine cervix: a combination of invasive margin pathological characteristics and lymphovascular space invasion. Asian Pac J Cancer Prev, 14, 6935-40.

Khunamornpong S, Settakorn J, Sukpan K, et al (2013). Prognostic value of pathological characteristics of invasive margins in early-stage squamous cell carcinomas of the uterine cervix. Asian Pac J Cancer Prev, 14, 5165-9.

Kim R, Emi M, Tanabe K (2007). Cancer immunoediting from immune surveillance to immune escape. Immunol, 121, 1-14.

Li H, Fan X, Houghton J (2007). Tumor microenvironment: the role of the tumor stroma in cancer. J Cell Biochem, 101, 805-15.

Liu J, Li J, Chen Y, et al (2014). Tumor-stroma ratio is an independent predictor for survival in early cervical carcinoma. Gynecol Oncol, 132, 81-6.
Mesker WE, Junggeburt JM, Szuhai K, et al (2007). The carcinoma-stromal ratio of colon carcinoma is an independent factor for survival compared to lymph node status and tumor stage. Cell Oncol, 29, 387-98.

Obel J, Souares Y, Hoy D, et al (2014). A systematic review of cervical cancer incidence and mortality in the Pacific Region. Asian Pac J Cancer Prev, 15, 9433-7.

Park JY, Kim DY, Kim JH, et al (2010). Outcomes after radical hysterectomy in patients with early-stage adenocarcinoma of uterine cervix. Br J Cancer, 102, 1692-8.

Peters WA, 3rd, Liu PY, Barrett RJ, 2nd, et al (2000). Concurrent chemotherapy and pelvic radiation therapy compared with pelvic radiation therapy alone as adjuvant therapy after radical surgery in high-risk early-stage cancer of the cervix. $J$ Clin Oncol, 18, 1606-13.

Pietras K, Ostman A (2010). Hallmarks of cancer: interactions with the tumor stroma. Exp Cell Res, 316, 1324-31.

Roma AA, Diaz De Vivar A, Park KJ, et al (2015). Invasive endocervical adenocarcinoma: a new pattern-based classification system with important clinical significance. Am J Surg Pathol, [Epub ahead of print].

Wang K, Ma W, Wang J, et al (2012). Tumor-stroma ratio is an independent predictor for survival in esophageal squamous cell carcinoma. J Thorac Oncol, 7, 1457-61.

West NP, Dattani M, McShane P, et al (2010). The proportion of tumour cells is an independent predictor for survival in colorectal cancer patients. Br J Cancer, 102, 1519-23.

Zhu QC, Gao RY, Wu W, et al (2013). Epithelial-mesenchymal transition and its role in the pathogenesis of colorectal cancer. Asian Pac J Cancer Prev, 14, 2689-98. 